\title{
Value of gamma interferon enzyme-linked immunospot assay in the diagnosis of peritoneal dialysis-associated tuberculous peritonitis
}

\author{
Qiuxia Fan ${ }^{1} \cdot$ Xiaoyan Huang $^{1} \cdot$ Jieyun Zhang $^{2} \cdot$ Yinan Sun $^{1} \cdot$ Zuying Xiong $^{1} \cdot$ Zibo Xiong $^{1}$
}

Received: 20 October 2020 / Accepted: 10 July 2021 / Published online: 14 July 2021

(c) The Author(s) 2021

\begin{abstract}
Background Tuberculous peritonitis is the most common form of extrapulmonary tuberculosis infection in peritoneal dialysis patients. However, diagnosing tuberculous peritonitis quickly and early has always been a challenge for nephrologists. Mycobacterium tuberculosis antigen-specific gamma interferon enzyme-linked immunospot (IFN- $\gamma$ ELISPOT) assay has been widely used in the clinical diagnosis of tuberculous pleurisy and peritonitis, but its use has not been reported for uremia. Methods This study mainly verified the feasibility of using the M. tuberculosis antigen-specific IFN- $\gamma$ ELISPOT assay in the diagnosis of continuous ambulatory peritoneal dialysis (CAPD) patients with tuberculous peritonitis. Taking $M$. tuberculosis culture as the gold standard, the IFN- $\gamma$ ELISPOT assay was used to analyze peripheral blood and peritoneal dialysis fluid of patients, and the receiver operating characteristic (ROC) curves in patients with tuberculous peritonitis (TBP) or non-tuberculous peritonitis (NTBP) were analyzed.

Results The area under the receiver operating characteristic curve (AUC) was 0.927 (95\% CI 0.816-1.000, $P=0.001$ ) for the ELISPOT assay with peritoneal fluid mononuclear cells (PFMC), which was higher than that for the ELISPOT assay with peripheral blood mononuclear cells (PBMC) $(0.825,95 \%$ CI $0.6490-1.000, P=0.011)$. The cutoff value for the diagnosis of TBP was 40 spot-forming cells (SFCs) $/ 2 \times 10^{5}$ for the ELISPOT with PBMC, with a sensitivity of $55.6 \%$, a specificity of $92.3 \%$, and a diagnostic efficiency of $77.3 \%$. The cutoff value for the diagnosis of TBP was $100 \mathrm{SFCs} / 2 \times 10^{5}$ for the ELISPOT on PFMC, with a sensitivity, specificity, and diagnostic efficiency $77.8 \%, 84.6 \%$, and $81.8 \%$, respectively. Parallel and serial testing algorithms appeared more accurate than single ELISPOT assays with PBMC, but ELISPOT assays with PFMC. Conclusions The IFN- $\gamma$ release test can be used for the early diagnosis of CAPD-related TBP; compared with peripheral blood, peritoneal fluid may be a more effective and accurate medium to diagnose CAPD complicated with tuberculous peritonitis.
\end{abstract}

Keywords Gamma interferon · Enzyme-linked immunospot experiments · Peritoneal dialysis · Tuberculous peritonitis

Qiuxia Fan and Xiaoyan Huang are contributed equally to this work.

Zibo Xiong

xiong0301@163.com

1 Renal Division, Peking University Shenzhen Hospital, Futian district, Lianhua road 1120, Shenzhen CN 518036, Guangdong, China

2 Guangdong Key Laboratory for Diagnosis and Treatment of Emerging Infectious Diseases, The Affiliated Shenzhen Third Hospital, Shenzhen 518020, China

\section{Introduction}

Patients with end-stage renal disease (ESRD) undergoing chronic dialysis are 6-25 times more likely to develop tuberculosis (TB) than the general population, mainly because of impaired cellular immunity, anemia, malnutrition, etc. [1-4]. Tuberculous peritonitis (TBP) is the most common form of extrapulmonary tuberculosis infection in peritoneal dialysis patients [5]. However, diagnosing active TB infection can be challenging. M. tuberculosis (liquid and solid) culture remains the gold standard diagnostic test, but it can take up to 10 weeks to become positive, introducing delays in diagnosis and treatment as well as associated increased morbidity [6]. Therefore, early and rapid diagnosis of tuberculous 
peritonitis has always been a challenge for nephrologists. T cell interferon gamma (IFN- $\gamma$ ) release assays (IGRAs) are now used to diagnose latent TB infection (LTBI) [7-9] and include a whole-blood gamma interferon (IFN- $\gamma$ ) enzymelinked immunosorbent assay (FERQuanti ON-TB Gold in-tube (QFT-G); Cellestis Ltd, Victoria, Australia) and an enzyme-linked immunospot (ELISPOT) assay (T-SPOT. TB; Oxford Immunotec, Oxfordshire, United Kingdom). $M$. tuberculosis antigen-specific gamma interferon (IFN- $\gamma$ ) production by peripheral blood mononuclear cells (PBMC) was determined by using an in-house enzyme-linked immunospot (ELISPOT) assay by Institute of Hepatology of Shenzhen Third Hospital [10]. The researchers developed and evaluated an in-house IFN- $\gamma$ ELISPOT assay for the diagnosis of active TB, compared the performance of their ELISPOT assay with that of the commercial QFT-IT assay and analyzed the influence of clinical manifestations on the accuracy of the ELISPOT assay. In this study, we evaluated the diagnostic performance of an M. tuberculosis antigen-specific IFN- $\gamma$ ELISPOT assay in continuous ambulatory peritoneal dialysis (CAPD) patients with tuberculous peritonitis.

\section{Materials and methods}

\section{Study population}

A cross-sectional study was performed, in which 22 patients with peritonitis were enrolled at Peking University Shenzhen
Hospital from January 2012 to December 2019. This study was approved by the Ethics Committee of Peking University Shenzhen Hospital. Written informed consent was obtained from all patients enrolled in this study. The incidence of peritonitis ranged from 0.102 to 0.225 episodes per patient years in our peritoneal dialysis (PD) center. The total number of peritonitis episodes in the center was 317 , and $M$. tuberculosis accounted for $2.84 \%$ of all peritonitis cases. A total of 308 episodes $(97.16 \%)$ had non-tuberculous peritonitis among all peritonitis cases. There were 33 patients with non-tuberculous peritonitis from January to December 2016. Of these patients, 20 patients were excluded from our study for the following reasons: antibiotics were used before taking samples $(n=11)$, treatment was provided in another center $(n=1)$, the patients were unwilling to keep specimens $(n=5)$, and data was unavailable $(n=3)$. Finally, 13 patients with bacterial or fungal peritonitis were enrolled as controls. Of the 13 patients with non-tuberculous peritonitis, 2 cases were caused by gram-positive bacteria, 4 were caused by gram-negative bacteria, and 3 were caused by fungi, and 4 patients had negative pathogenic bacterial cultures and were cured after routine antibiotic treatment (ceftazidime combined with cefazolin). The flow chart of the study population is shown in Fig. 1. Patients with HIV infection and active tuberculosis in other locations were excluded.

\section{Diagnostic criteria}

Peritoneal dialysis-related peritonitis was always diagnosed when at least 2 of the following were present: (1) clinical
Fig. 1 Flow chart of the study population. $T B P$ tuberculous peritonitis, $N T B P$, nontuberculous peritonitis, $P B M C$ peripheral blood mononuclear cells, $P F M C$ peritoneal fluid mononuclear cells

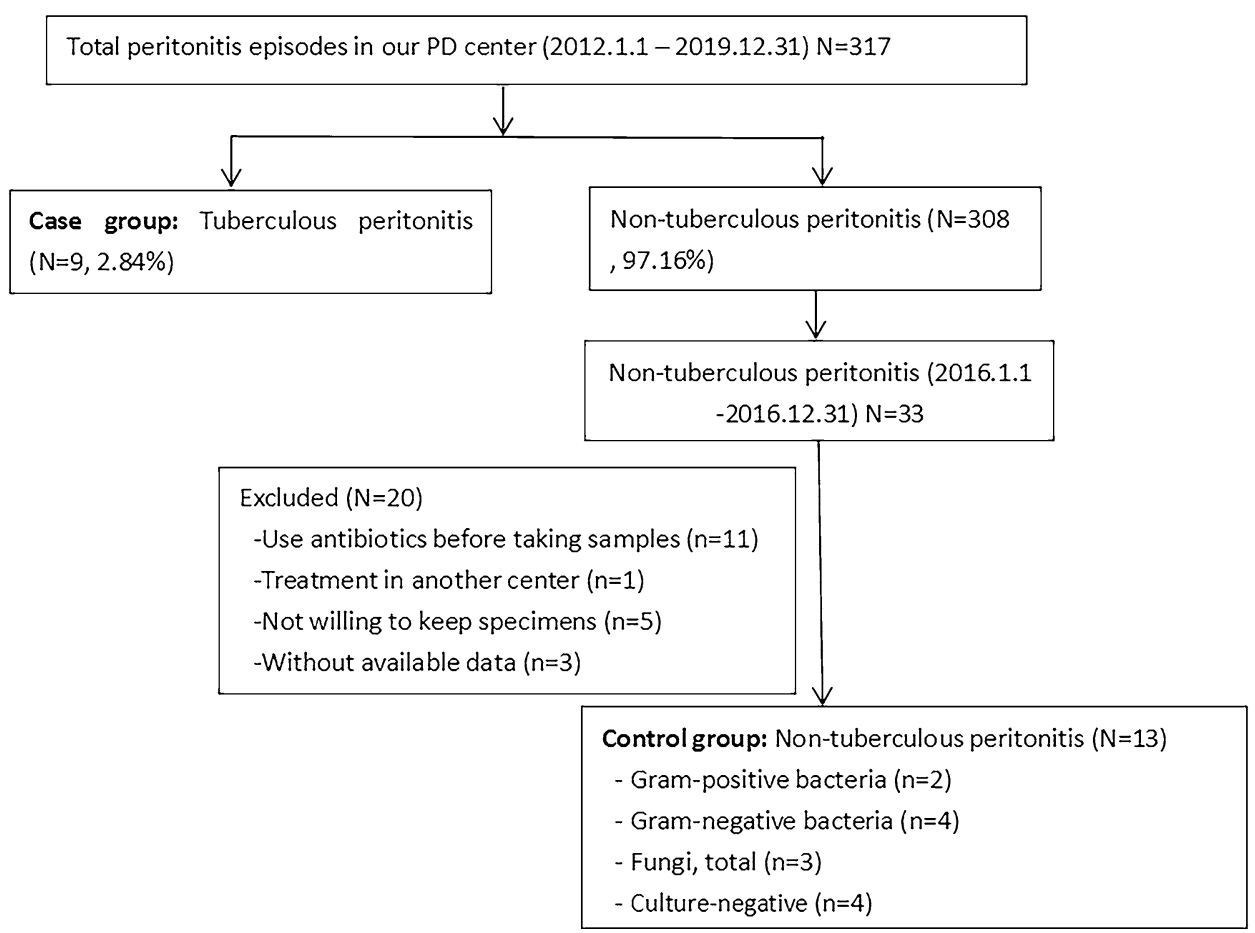


features consistent with peritonitis, i.e., abdominal pain and/or cloudy dialysis effluent; (2) dialysis effluent white cell count $>100 / \mu \mathrm{L}$ or $>0.1 \times 10^{9} / \mathrm{L}$ (after a dwell time of at least $2 \mathrm{~h}$ ), with $>50 \%$ polymorphonuclear cells; and (3) positive dialysis effluent culture. The diagnosis of tuberculous peritonitis was confirmed if a patient's dialysis effluent was positive for M. tuberculosis culture. According to symptoms, physical signs, the clinical course, and laboratory and imaging examination findings, patients with highly suspected tuberculous peritonitis could also be diagnosed if their PD effluent was culture-negative and improved after anti-tuberculosis treatment [11].

\section{PBMC and PFMC ELISPOT assays}

Peripheral blood and peritoneal fluid were obtained before the treatment of peritonitis patients, at this time, patients often have clinical manifestations such as abdominal pain, fever, cloudy dialysis effluent, and excessive number of peritoneal fluid cells. Ten milliliters of peripheral blood were collected from the participants, and peripheral blood mononuclear cells (PBMC) were obtained from whole blood by centrifugation over a Ficoll Hypaque density gradient (Ficoll-Paque Plus; Amersham Biosciences). Cells were resuspended in Lympho-Spot medium (U-CyTech Bioscience, the Netherlands). At the same time, $50-100 \mathrm{~mL}$ of peritoneal fluid that remained within the intraperitoneal cavity for no less than $2 \mathrm{~h}$ was collected to obtain PFMC by centrifugation at $300 \times g$ for $5 \mathrm{~min}$. It was necessary to reach $2 \times 10^{5} \mathrm{PFMC} /$ well for a total of four wells, and the number of monocytes required was $8 \times 10^{5}$, which could be obtained by centrifugation of $50-100 \mathrm{~mL}$ of peritoneal dialysis fluid. The sediment was suspended in $5 \mathrm{~mL}$ of red blood cell lysis solution (MiltenyiBiotec GmbH, Germany), allowed to stand for $1 \mathrm{~min}$ at room temperature, and then mixed with $5 \mathrm{~mL}$ of RPMI 1640 containing 10\% fetal bovine serum (FBS). This suspension was centrifuged at $300 \times g$ for $5 \mathrm{~min}$, and the cells were resuspended in $5 \mathrm{~mL}$ of RPMI 1640 containing 10\% FBS. Ten microliters of this suspension were mixed with $90 \mu \mathrm{L}$ of trypan blue solution to evaluate the viability and to count cells. The cells were resuspended in Lympho-Spot medium (U-CyTech Biosciences, the Netherlands) at a final concentration of $2 \times 10^{6}$ cells $/ \mathrm{mL}$. PBMC or PFMC $\left(2 \times 10^{5} \%\right.$ well) were seeded in duplicate in 96-well plates (MultiScreen-IP; Millipore) precoated with an anti-IFN- $\gamma$ capture monoclonal antibody (eBioscience). Cells were stimulated with the Mycobacterium tuberculosis-specific antigen early secretory antigenic target 6 (ESAT-6) protein (provided by Haiying Liu, Chinese Academy of Medical Sciences) at $10 \mu \mathrm{g} / \mathrm{mL}$ for $24 \mathrm{~h}$ at $37{ }^{\circ} \mathrm{C}$ and with $5 \% \mathrm{CO}_{2}$. PBMC and PFMC in medium alone or stimulated with phytohemagglutinin (Sigma) at $2.5 \mu \mathrm{g} / \mathrm{mL}$ were used as negative or positive controls, respectively. Biotinylated anti-IFN- $\gamma$ detection monoclonal antibodies (eBioscience) were added for $1 \mathrm{~h}$, followed by the addition of streptavidin-alkaline phosphatase conjugate (Pierce Biotechnology), which was incubated for $1 \mathrm{~h}$. After a wash step, tetrazolium-BCIP (5-bromo-4-chloro3-indolyl phosphate; Sigma, USA) chromogenic substrate was added. The individual spots were counted using an automated image analysis ELISPOT reader system (BioReader 4000 Pro-X; Biosys, Germany) [10, 12, 13].

\section{Statistical analysis}

Statistical analyses were performed using IBM SPSS for Windows, version 23.0 (SPSS); the MedCalc software package, version 18.2.1.0 (MedCalc Software); and GraphPad Prism, version 8.0.1 (GraphPad, La Jolla, CA). Continuous variables were compared using the Mann-Whitney $U$ test or Student's $t$ test, and Fisher's exact test was used to compare categorical variables. $P$ values $<0.05$ were considered significant. Diagnostic performance was expressed in terms of sensitivity, specificity, positive predictive value, negative predictive value, positive likelihood ratio, and negative likelihood ratio.

\section{Results}

\section{Patient characteristics}

A total of 22 patients with peritonitis were qualified and recruited for this study. Nine patients were diagnosed with TBP. Among them, 8 were positive, and 1 was negative in dialysate by $M$. tuberculosis culture; the patient with a negative culture was diagnosed by clinical manifestations, and effective anti-tuberculosis treatment was performed after 2-4 weeks. The remaining 13 patients were confirmed to have peritonitis that were not caused by M. tuberculosis infection (NTBP). The clinical characteristics of the patients are summarized in Table 1. Overall, there were significant differences in the spot number of ESAT-6-induced IFN- $\gamma$ production in peritoneal fluid and the spot number in peripheral blood, and peritoneal fluid was 4-9 times higher than that in peripheral blood. There was a higher percentage of TB patients with a previous infection history than of NTB patients, and there was no difference in serum creatinine, blood urea nitrogen, albumin (ALB) or hemoglobin (HGB) levels.

\section{Results of ELISPOT assays}

ELISPOT assays using PBMC and PFMC were performed on samples from all 22 subjects; $5(55.6 \%)$ out of 9 patients with TBP were positive by the PBMC ELISPOT assay, and 7 (77.8\%) out of 9 were positive by the PFMC ELISPOT 
Table 1 Baseline clinical characteristics of peritoneal dialysis-associated peritonitis patients

\begin{tabular}{|c|c|c|c|}
\hline Characteristic & $\begin{array}{l}\text { TBP } \\
(n=9)\end{array}$ & $\begin{array}{l}\text { NTBP } \\
(n=13)\end{array}$ & $P$ value \\
\hline Gender ( $\%$ male) & $7(77.8 \%)$ & $4(30.8 \%)$ & $0.080^{\mathrm{b}}$ \\
\hline Age (years) & $57 \pm 11.44$ & $56.54 \pm 14.85$ & $0.938^{\mathrm{a}}$ \\
\hline PD duration (years) & $3.06 \pm 2.38$ & $3.63 \pm 2.68$ & $0.611^{\mathrm{a}}$ \\
\hline Diabetes mellitus (\%) & $3(33.3 \%)$ & $2(15.4 \%)$ & $0.323^{\mathrm{b}}$ \\
\hline Previous history of TB (\%) & $4(44.4 \%)$ & $0(0 \%)$ & $0.017^{\mathrm{b}}$ \\
\hline The main leukocytes from PF are mononuclear cells (\%) & $2(22.2 \%)$ & $0(0 \%)$ & $0.156^{\mathrm{b}}$ \\
\hline ESAT-6-induced IFN- $\gamma$ production from PB $\left(\mathrm{SFC} / 2 \times 10^{5} \mathrm{PBMC}\right)$ & $41(17.5-91)$ & $8(2.5-36)$ & $0.011^{\mathrm{c}}$ \\
\hline ESAT-6-induced IFN- $\gamma$ production from $\mathrm{PF}\left(\mathrm{SFC} / 2 \times 10^{5} \mathrm{PBMC}\right)$ & $385(201-393)$ & $35(2-54)$ & $0.001^{\mathrm{c}}$ \\
\hline WBC from $\mathrm{PB}\left(\times 10^{9} / \mathrm{L}\right)$ & $7.9 \pm 4.68$ & $9.17 \pm 3.76$ & $0.491^{\mathrm{a}}$ \\
\hline WBC from $P F\left(\times 10^{6} / \mathrm{L}\right)$ & $315(113-735)$ & $1270(166-6483.5)$ & $0.095^{\mathrm{c}}$ \\
\hline Hemoglobin $(g / L)$ & $99.0 \pm 23.7$ & $93.62 \pm 15.5$ & $0.525^{\mathrm{a}}$ \\
\hline Serum creatinine $(\mu \mathrm{mol} / \mathrm{L})$ & $810(757.65-1097.4)$ & $715(620-825)$ & $0.089^{c}$ \\
\hline Blood urea nitrogen (mmol/L) & $15.15 \pm 1.60$ & $12.83 \pm 4.35$ & $0.144^{\mathrm{a}}$ \\
\hline Calcium and phosphorus product $\left(\mathrm{mg}^{2} / \mathrm{dL}^{2}\right)$ & $33.97(29.12-47.63)$ & $32.1(26.64-44.4)$ & $0.483^{\mathrm{c}}$ \\
\hline Albumin (g/L) & $27.50 \pm 6.15$ & $27.27 \pm 5.33$ & $0.954^{\mathrm{a}}$ \\
\hline Parathyroid hormone (pmol/L) & $18.2(11.3-31)$ & $19.7(10.1-42.7)$ & $0.92^{\mathrm{c}}$ \\
\hline
\end{tabular}

$T B$ tuberculosis, $P B M C$ peripheral blood mononuclear cells, $P F M C$ peritoneal fluid mononuclear cells

${ }^{a}$ Independent sample $t$ test

${ }^{\mathrm{b}}$ Fisher exact test

${ }^{\mathrm{c}}$ Mann-Whitney test

assay. Among the controls, the ELISPOT assay was positive for one $(7.7 \%)$ out of 13 NTBP patients when performed on PBMC and two (15.4\%) out of 13 when performed on PFMC. The numbers of SFCs in response to ESAT-6 protein among PBMC are in good correlation with those among PFMC $\left(r_{\mathrm{s}}=0.579, P=0.005\right)$, indicating that the protein can efficiently stimulate IFN- $\gamma$ production in an ELISPOT assay in both PBMC and in PFMC. We found that the levels of antigen-specific IFN- $\gamma$-producing cells were significantly increased among PFMC compared with those among matched PBMC in TBP patients, the median were 385 and $41 \mathrm{SFCs} / 2 \times 10^{5}$, respectively $(P=0.005)$. As expected, the numbers of antigen-specific IFN- $\gamma$-producing cells were not different between PFMC and PBMC from NTBP patients, the median were 35 and $8 \mathrm{SFCs} / 2 \times 10^{5}$, respectively $(P=0.292)$ (Fig. 2).

Receiver operating characteristic (ROC) analysis demonstrated that PFMC ELISPOT assays had higher areas under the curve (AUCs) than PBMC ELISPOT assays (Fig. 3). The AUC was 0.927 (95\% CI 0.816-1.000, $P=0.001$ ) for the ELISPOT assay with PFMC, which was higher than that for the ELISPOT on PBMC $(0.825,95 \%$ CI $0.6490-1.000$, $P=0.011)$. The cutoff value for the diagnosis of TBP was 40 spot-forming cells (SFCs) $/ 2 \times 10^{5}$ for the ELISPOT assay with PBMC, with a sensitivity of $55.6 \%$, a specificity of $92.3 \%$, and a diagnostic efficiency of $77.3 \%$. The positive predictive value was $83.3 \%$, with a negative predictive value

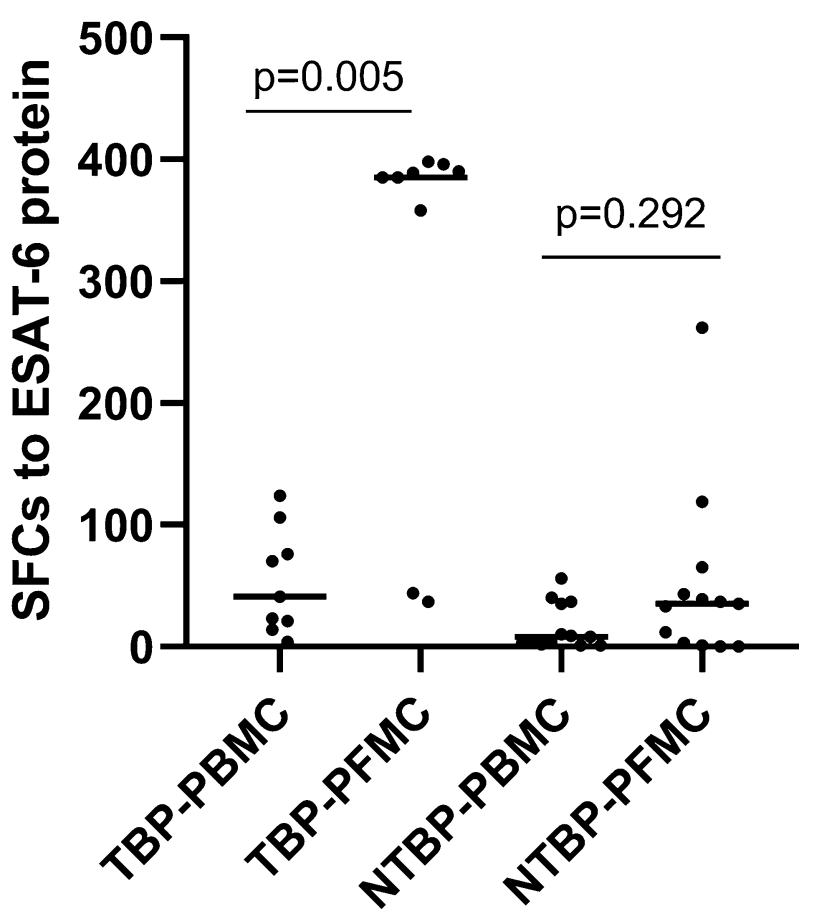

Fig. 2 M. tuberculosis antigen-specific IFN- $\gamma$ responses are enriched in PFMC over PBMC from patients with TBP. IFN- $\gamma$ responses to ESAT-6 protein were determined in PBMC and PFMC from patients with TBP and from NTBP patients. Each spot represents an individual data point. SFCs spot-forming cells, ESAT-6 protein mycobacterium tuberculosis-specific antigen early secretory antigenic target 6 


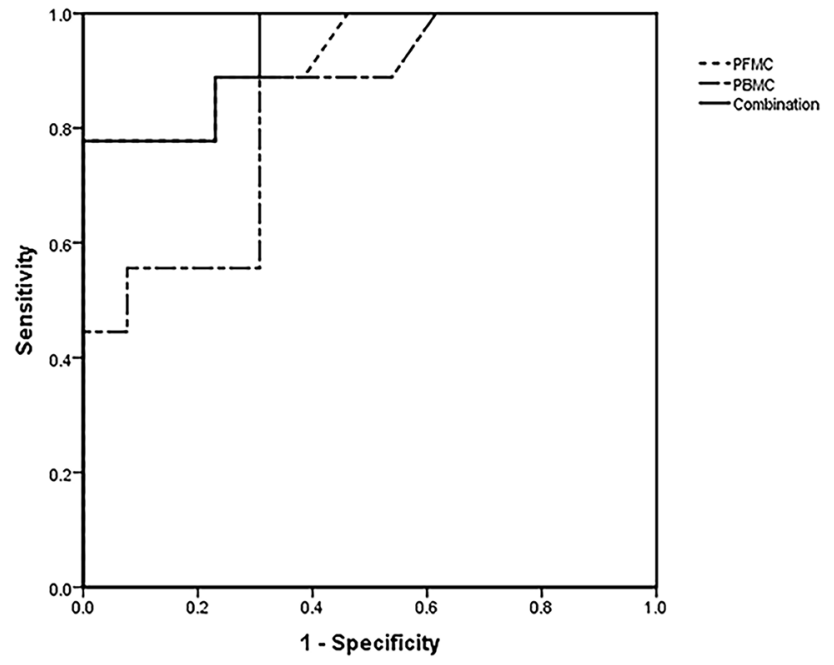

Fig. 3 ROC curves for the ELISPOT assays with PFMC and PBMC from patients with tuberculous peritonitis or non-tuberculous peritonitis. The M. tuberculosis antigen-specific IFN- $\gamma$ response in PFMC ELISPOT assays is useful for the diagnosis of TBP. The AUC was 0.927 (95\% CI $0.816-1.000, P=0.001)$ for the ELISPOT assay with PFMC, which was higher than that of the ELISPOT assay with PBMC $(0.825,95 \%$ CI $0.6490-1.000, P=0.011)$. The AUC of the combination of PBMC and PFMC increased the c-statistic from 0.927 to 0.94 . $P B M C$ peripheral blood mononuclear cells, $P F M C$ peritoneal fluid mononuclear cells, $R O C$ receiver operating characteristic, $A U C$ area under the receiver operating characteristic curve. The AUC of the combination of the ELISPOT assays with PFMC and PBMC to diagnose TBP was calculated with ROC curve analysis. The cutoff values of PFMC and PBMC in identifying TBP were 100 and 40 $\mathrm{SFCs} / 2 \times 10^{5}$, respectively, which were obtained according to Chen's study

of $75 \%$, a positive likelihood ratio of 7.2 , and a negative likelihood ratio of 0.48 . The cutoff value for the diagnosis of TBP was $100 \mathrm{SFCs} / 2 \times 10^{5}$ for the ELISPOT assay with PFMC, with sensitivity, specificity, diagnostic efficiency, PPV, NPV, LR +, and LR - of 77.8\%, 84.6\%, 81.8\%, 77.8\%, $84.6 \%, 5.05$ and 0.26 , respectively. Thus, the performance of the PFMC ELISPOT assay for the diagnosis of TBP is significantly better than that of the PBMC ELISPOT assay. Parallel and serial testing algorithms appeared more accurate than the single ELISPOT assay with PBMC, but ELISPOT assay with PFMC. The parallel testing increased the sensitivity of the ELISPOT assay with PFMC from 77.8 to $88.9 \%$, but the specificity was decreased from 84.6 to $76.9 \%$. Serial testing increased the specificity of the ELISPOT assay with PFMC from 87.1 to $100 \%$, but the sensitivity was decreased from 55.6 to $44.4 \%$ (Table 2).

\section{Discussion}

Peritoneal dialysis-related tuberculous peritonitis is the most common form of extrapulmonary tuberculosis infection in CAPD patients [5]. Diagnosis can often be challenging, due to the indolent nature of the presentation of TB peritonitis and relative unfamiliarity with the infection, and even when clinically suspected, diagnosing active TB infection is not easy. Mycobacterial culture remains the gold standard laboratory diagnostic method for TB peritonitis, but even with modern liquid culture techniques, a positive result may require up to 10 weeks of incubation [6]. The positive period of tuberculosis culture dialysis fluid of 8 cases was 6-10 weeks. Second, the acid-fast bacillus smear of peritoneal dialysis fluid is not sensitive. Only 2 of 9 cases of TBP were positive for acid-fast staining, accounting for $22.2 \%$, so the rate of missed diagnosis was high. Furthermore, due to the low delayed hypersensitivity in ESRD patients, the positive rate of the tuberculin purified protein derivative (PPD) test in dialysis patients was $15 \%$ on average. Due to extensive vaccination with the Bacillus Calmette-Guerin (BCG) vaccine, the PPD test has lost its diagnostic value and has no reference value in dialysis patients [14]. Additionally, the clinical symptoms of TBP in CAPD are fever, abdominal pain and turbid peritoneal dialysate, which are similar to those of non-tuberculous peritonitis and are not typical. This delay in diagnosis and treatment can result in increased morbidity and may have infection control implications. A delay in diagnosis in the presence of persistent symptoms may mean that the PD catheter has to be removed, adding to the treatment burden of the patient having to convert to hemodialysis. Therefore, it is difficult to diagnose and treat TBP at this stage, and the mortality rate is much higher than

Table 2 Diagnostic accuracy estimates for the ESAT-6 interferon- $\gamma$ ELISPOT assay with PFMC and PBMC

\begin{tabular}{lllllrrr}
\hline & Sensitivity, $\%$ & Specificity, $\%$ & $\begin{array}{l}\text { Diagnostic } \\
\text { efficiency, } \%\end{array}$ & PPV, \% & NPV, \% & LR+ \\
\hline ELISPOT with PFMC & 77.8 & 84.6 & 81.8 & 77.8 & 84.6 & 5.05 & 0.26 \\
ELISPOT with PBMC & 55.6 & 92.3 & 77.3 & 83.3 & 75.0 & 7.20 & 0.48 \\
ELISPOT with PBMC \& PFMC (parallel) & 88.9 & 76.9 & 81.8 & 72.7 & 90.9 & 3.85 & 0.14 \\
ELISPOT with PBMC \& PFMC (serial) & 44.4 & 100 & 77.3 & 100 & 72.0 & N/A & 0.56 \\
\hline
\end{tabular}

$P P V$ positive predictive value, $N P V$ negative predictive value, $L R+$ positive likelihood ratio, $L R$ - negative likelihood ratio, $P B M C$ peripheral blood mononuclear cells, $P F M C$ peritoneal fluid mononuclear cells, N/A not applicable 
that of non-tuberculosis peritonitis [15]. The best way to improve the survival rate of patients is to find an effective method for the early diagnosis of TBP and provide timely treatment.

In 2009, Chen, Xinchun et al. developed an in-house IFN- $\gamma$ enzyme-linked immunospot (ELISPOT) assay and evaluated its value in the diagnosis of active tuberculosis (TB) in Shenzhen, China [10]. Their study demonstrates that the IFN- $\gamma$ ELISPOT assay is a useful adjunct to current tests for the diagnosis of active TB in China. They developed an independent antigen-specific IFN- $\gamma$ ELISPOT kit for tuberculosis bacteria. Compared with results from a QFT-G kit, the positive rates were $75.0 \%$ and $78.1 \%$, and the difference was insignificant $(P>0.05)$. The positive threshold of ESAT-6 in peripheral blood was $40 \mathrm{SFCs} / 2 \times 10^{5}$. In our study, the optimal critical value of ESAT-6-induced spots in peripheral blood was $40 \mathrm{SFCs} / 2 \times 10^{5}$, and the sensitivity and specificity were $85.7 \%$ and $84.6 \%$, respectively, which were close to the values reported in Chen's study. This finding shows that the uremic population and the general population have the same diagnostic threshold for peripheral blood.

The early diagnosis of tuberculosis infection by IGRAs is mainly based on the detection of peripheral blood antigenstimulated polymorphonuclear cells. However, the detection with peripheral blood cannot effectively distinguish active TB from latent TB. In CAPD patients with tuberculous peritonitis, the number of leukocytes in the exudate increased significantly. It is feasible to use exudate monocytes to assist in the diagnosis of tuberculous peritonitis. The design theory of this experiment explored the feasibility of exudate monocytes in an ELISPOT to assist in the diagnosis of CAPD patients with tuberculous peritonitis. Wilkinson KA et al. first proposed the feasibility of using ELISPOT assays with monocytes from pleural and ascitic fluid to diagnose tuberculous serositis. In patients with tuberculosis, the mean concentration $( \pm$ SD) of antigen-specific, IFN- $\gamma$-producing SFCs in the ascitic fluid was approximately 17 -fold \pm sixfold greater than that in peripheral blood [16]. In our study, we also found that the number of IFN- $\gamma$-specific T cells in the peritoneal dialysis fluid of patients with tuberculous peritonitis was 4-9 times higher than that in their peripheral blood.

Previous studies by our collaborators found that when the detection threshold of a TB-specific IFN-gamma ELISPOT in pleural effusion was $100 \mathrm{SFCs} / 2 \times 10^{5}$, the sensitivity and specificity for the diagnosis of tuberculous pleurisy were $95.7 \%$ and $100 \%$, respectively [13]. Sung-Han Kim et al. demonstrated that an ELISPOT assay using PBMC and PFMC is a useful adjunct to the current tests for diagnosing abdominal TB [17]. One possible explanation for the results is that an inflamed serosal surface may inevitably allow circulating $\mathrm{M}$. tuberculosis-specific lymphocyte migration in patients with inactive TB and latent TB infection. In our study, when the cutoff value of the recombinant antigen ESAT-6 in dialysis fluid was $100 \mathrm{SFCs} / 2 \times 10^{5}$, the diagnostic sensitivity was $77.8 \%$, and the specificity was $84.6 \%$. The AUC for the ELISPOT assay with PFMC was higher than that of the ELISPOT assay with PBMC, and the AUC of the combination of PBMC and PFMC increased the c-statistic from 0.93 to 0.94 . Therefore, compared with peripheral blood, peritoneal dialysate may be a more effective and accurate medium to diagnose CAPD complicated with tuberculous peritonitis, and the combination of peritoneal dialysate and peripheral blood may also be an effective choice. The ELISPOT technique for peritoneal dialysis fluid is expected to be a rapid method for the clinical diagnosis of tuberculous peritonitis, and it is worth popularizing and applying widely.

Whether catheter removal is necessary for tuberculous peritonitis is still controversial. The 2016 ISPD guidelines tend to support the notion of extubation when $M$. tuberculosis peritonitis occurs and to consider reintroduction after 6 weeks of anti-tuberculosis treatment. With an increasing number of reported cases without extubation, some scholars believe that peritoneal function is an important condition to consider to determine whether patients continue peritoneal dialysis, and studies have proven that tuberculosis bacteria cannot survive in peritoneal dialysis catheters. If patients can maintain a certain level of ultrafiltration and transport function, they may not be extubated. Tuberculosis infection is not a sufficient condition to terminate peritoneal dialysis [18]. In this study, 2 patients were not extubated and continued undergoing peritoneal dialysis, which also supports this point of view.

Our study has several strengths and shortcomings. The major strengths of this study include that we enrolled nine tuberculosis peritonitis patients in 8 years, which is not easy because CAPD-related TBP is a rare disease. Moreover, tuberculosis culture was used as the golden standard in diagnosing tuberculosis peritonitis, all patients were followed-up, and prognosis at a specific period was observed in our study. However, shortcomings should also be considered. Major limitations of this study include that the study is a single-center, small-sample study; we look forward to the production of multicenter, large-sample data for further confirmation. In addition, there are some deficiencies in this technique. For example, although ESAT-6 is absent in all BCG strains and most environmental mycobacteria, the positive results of the ELISPOT assay may be caused by the infection of Mycobacterium kansaii, Mycobacterium simiae, Mycobacterium gordonae or Mycobacterium marinum. It should be screened in clinical diagnosis.

\section{Conclusions}

In conclusion, the IFN-gamma release test can be used for the early diagnosis of CAPD-related TBP; compared with peripheral blood, peritoneal dialysate may be a more 
effective and accurate medium to diagnose CAPD complicated with tuberculous peritonitis.

Acknowledgements We thank Jieyun Zhang from Guangdong Key Laboratory for Diagnosis \& Treatment of Emerging Infectious Diseases, the Affiliated Shenzhen Third Hospital for her technical assistance. This study was supported by the Shenzhen Sanming Project (SZSM201812097).

Author contributions ZBX designed the study; QXF, JYZ and YNS collected data and followed up the patients; QXF, ZBX, XYH, and ZYX analyzed the data; QXF made the figures; ZBX and QXF drafted and revised the paper; all authors approved the final version of the manuscript.

\section{Declarations}

Conflict of interest The authors declare that they have no conflicts of interest.

Open Access This article is licensed under a Creative Commons Attribution 4.0 International License, which permits use, sharing, adaptation, distribution and reproduction in any medium or format, as long as you give appropriate credit to the original author(s) and the source, provide a link to the Creative Commons licence, and indicate if changes were made. The images or other third party material in this article are included in the article's Creative Commons licence, unless indicated otherwise in a credit line to the material. If material is not included in the article's Creative Commons licence and your intended use is not permitted by statutory regulation or exceeds the permitted use, you will need to obtain permission directly from the copyright holder. To view a copy of this licence, visit http://creativecommons.org/licenses/by/4.0/.

\section{References}

1. Segall L, Covic A (2010) Diagnosis of tuberculosis in dialysis patients: current strategy. Clin J Am Soc Nephrol 5:1114-1122

2. Wauters A, Peetermans WE, Van den Brande P et al (2004) The value of tuberculin skin testing in haemodialysis patients. Nephrol Dial Transplant 19:433-438

3. Habesoglu MA, Torun D, Demiroglu YZ et al (2007) Value of the tuberculin skin test in screening for tuberculosis in dialysis patients. Transplant Proc 39:883-886

4. Passalent L, Khan K, Richardson R, Wang J, Dedier H, Gardam M (2007) Detecting latent tuberculosis infection in hemodialysis patients: a head-to-head comparison of the T-SPOT.TB test, tuberculin skin test, and an expert physician panel. Clin J Am Soc Nephrol 2:68-73
5. Vaid U, Kane G (2017) Tuberculous peritonitis. Microbiol spectr. https://doi.org/10.1128/microbiolspec.TNMI7-0006-2016

6. Edwards S, Glynn P, David MD, Kamesh L (2016) Diagnosing tuberculous peritonitis early in patients on peritoneal dialysis: use of xpert MTB/RIF assay. Perit Dial Int 36:461-463

7. Adetifa IM, Lugos MD, Hammond A et al (2007) Comparison of two interferon gamma release assays in the diagnosis of mycobacterium tuberculosis infection and disease in the gambia. BMC Infect Dis 7:122

8. Ferguson TW, Tangri N, Macdonald K et al (2015) The diagnostic accuracy of tests for latent tuberculosis infection in hemodialysis patients: a systematic review and meta-analysis. Transplantation 99:1084-1091

9. Haas MK, Belknap RW (2019) Diagnostic tests for latent tuberculosis infection. Clin Chest Med 40:829-837

10. Chen X, Yang Q, Zhang M et al (2009) Diagnosis of active tuberculosis in China using an in-house gamma interferon enzymelinked immunospot assay. Clin Vaccine Immunol 16:879-884

11. Li PK, Szeto CC, Piraino B et al (2016) ISPD peritonitis recommendations: 2016 update on prevention and treatment. Perit Dial Int 36:481-508

12. Zhang MWH, Liao M, Chen X, Graner M, Zhu X, Zhang J, Yang Q, Lu H, Zhou BCH (2010) Diagnosis of latent tuberculosis infection in bacille Calmette-Guérin vaccinated subjects in China by interferon-gamma ELISpot assay. Int J Tuberc Lung Dis 14(12):1556-1563

13. Liao M, Yang Q, Zhang J et al (2014) Gamma interferon immunospot assay of pleural effusion mononuclear cells for diagnosis of tuberculous pleurisy. Clin Vaccine Immunol 21:347-353

14. Smirnoff M, Patt C, Seckler B, Adler JJ (1998) Tuberculin and anergy skin testing of patients receiving long-term hemodialysis. Chest 113:25-27

15. Talwani R, Horvath JA (2000) Tuberculous peritonitis in patients undergoing continuous ambulatory peritoneal dialysis: case report and review. Clin Infect Dis 31:70-75

16. Wilkinson KA, Wilkinson RJ, Pathan A et al (2005) Ex vivo characterization of early secretory antigenic target 6 -specific $\mathrm{T}$ cells at sites of active disease in pleural tuberculosis. Clin Infect Dis 40:184-187

17. Kim SH, Cho OH, Park SJ et al (2009) Diagnosis of abdominal tuberculosis by T-cell-based assays on peripheral blood and peritoneal fluid mononuclear cells. J Infect 59:409-415

18. Lui SL, Chan TM, Lai KN, Lo WK (2007) Tuberculous and fungal peritonitis in patients undergoing continuous ambulatory peritoneal dialysis. Perit Dial Int 27(Suppl 2):S263-266

Publisher's Note Springer Nature remains neutral with regard to jurisdictional claims in published maps and institutional affiliations. 